\title{
Improved left ventricular unloading and circulatory support with synchronized pulsatile left ventricular assistance compared with continuous-flow left ventricular assistance in an acute porcine left ventricular failure model
}

\author{
George V. Letsou, MD, ${ }^{\mathrm{a}}$ Thomas D. Pate, MS, ${ }^{\mathrm{d}}$ Jeffrey R. Gohean, MS, ${ }^{\mathrm{d}}$ Mark Kurusz, BA, CCP ${ }^{\mathrm{d}}$ \\ Raul G. Longoria, $\mathrm{PhD},{ }^{\mathrm{c}}$ Larry Kaiser, $\mathrm{MD},{ }^{\mathrm{b}}$ and Richard W. Smalling, $\mathrm{MD}, \mathrm{PhD}^{\mathrm{a}}$
}

\begin{abstract}
Objective: Controversy exists regarding the optimal pumping method for left ventricular assist devices. The purpose of this investigation was to test the hypothesis that pulsatile left ventricular assist synchronized to the cardiac cycle provides superior left ventricular unloading and circulatory support compared with continuous-flow left ventricular assist devices at the same level of ventricular assist device flow.
\end{abstract}

\begin{abstract}
Methods: Seven male pigs were used to evaluate left ventricular assist device function using the TORVAD synchronized pulsatile-flow pump (Windmill Cardiovascular Systems, Inc, Austin, Tex) compared with the Bio-Medicus BPX-80 continuous-flow centrifugal pump (Medtronic, Inc, Minneapolis, Minn). Experiments were carried out under general anesthesia, and animals were instrumented via a median sternotomy. Hemodynamic measurements were obtained in the control state and with left ventricular assistance using the TORVAD and BPX-80 individually. Left ventricular failure was induced with suture ligation of the mid-left anterior descending coronary artery, and hemodynamic measurements were repeated.
\end{abstract}

Results: During left ventricular assist device support, mean aortic pressure and total cardiac output were higher and left atrial pressure was lower with pulsatile compared with continuous flow at the same ventricular assist device flow rate. During ischemic left ventricular failure, pulsatile left ventricular support resulted in higher total cardiac output $(5.58 \pm 1.58$ vs $5.12 \pm 1.19, P<.05)$, higher mean aortic pressure $(67.8 \pm 14$ vs $60.2 \pm 10$, $P<.05)$, and lower left atrial pressure $(11.5 \pm 3.5$ vs $13.9 \pm 6.0, P<.05)$ compared with continuous flow at the same left ventricular assist device flow rate.

Conclusion: Synchronized, pulsatile left ventricular assistance produces superior left ventricular unloading and circulatory support compared with continuous-flow left ventricular assist at the same flow rates. (J Thorac Cardiovasc Surg 2010;140:1181-8)

Pulsatile left ventricular assist devices (LVADs) have inlet and outlet valves with areas of stasis associated with embolization and increased risk of stroke. ${ }^{1}$ They are also prone to mechanical failure, typically do not synchronize with the cardiac cycle, and have fixed stroke volumes. ${ }^{2}$

\footnotetext{
From the Divisions of Cardiovascular Medicine ${ }^{\mathrm{a}}$ and Cardiothoracic Surgery, ${ }^{\mathrm{b}}$ The University of Texas Medical School at Houston and The Memorial Hermann Heart and Vascular Institute, Houston, Tex; Department of Mechanical Engineering, ${ }^{\mathrm{c}}$ The University of Texas at Austin, Austin, Tex; and Windmill Cardiovascular Systems, Inc, ${ }^{\mathrm{d}}$ Austin, Tex.

Disclosures: Supported in part by Windmill CV Systems. Mark Kurusz reports consulting fees and equity ownership of Windmill CV. Raul G. Longoria reports consulting fees, equity ownership, and gift support from Windmill CV, and holds patents related to the TORVAD device used in this study. Thomas D. Pate reports equity ownership and is an employee of Windmill CV. Richard W. Smalling reports equity ownership of Windmill CV.

Received for publication Dec 5, 2009; revisions received March 3, 2010; accepted for publication March 20, 2010; available ahead of print May 24, 2010.

Address for reprints: Richard W. Smalling, MD, PhD, Department of Internal Medicine, Division of Cardiovascular Medicine, University of Texas Houston-Medical School, 6431 Fannin, MSB1.246, Houston, TX 77030 (E-mail: richard.w. smalling@uth.tmc.edu).

$0022-5223 / \$ 36.00$

Copyright (c) 2010 by The American Association for Thoracic Surgery

doi:10.1016/j.jtcvs.2010.03.043
}

In contrast, continuous-flow LVADs do not require valves and their size is less bulky, but they also cannot synchronize with the cardiac cycle and flow rates vary with preload and after-load for a given pump speed. ${ }^{3,4}$ At maximum flow rates, the aortic valve may not open, leading to potential fusion of the aortic leaflets and thrombus formation in the sinus of Valsalva, potentially increasing the risk of cerebral embolization. ${ }^{5}$ In addition, aortic leaflet fusion can induce aortic insufficiency and lead to the need for increased VAD support, making weaning less likely. ${ }^{6}$ Thrombus formation has also been reported within the pumping mechanism of continuous-flow devices. ${ }^{7}$

Continuous-flow devices are frequently associated with absence of detectable pulses in patients with severe left ventricular (LV) dysfunction, making determination of true arterial blood pressure difficult. ${ }^{8}$ This makes it difficult to up-titrate after-load reducing agents to ameliorate congestive heart failure symptoms and improve the chances for LV recovery. ${ }^{1,3}$

The TORVAD (Windmill Cardiovascular Systems, Inc, Austin, Tex) LVAD is a valveless, pulsatile, rotary, dualpiston, positive displacement pump with a controller, power/sensor cable, and power supply. The pump performs 


$$
\begin{aligned}
& \text { Abbreviations and Acronyms } \\
& \text { AoP }=\text { aortic pressure } \\
& \text { BTT }=\text { bridge to transplant } \\
& \text { ECG }=\text { electrocardiogram } \\
& \text { IV }=\text { intravenous } \\
& \text { LAD }=\text { left anterior descending } \\
& \text { LAP }=\text { left atrial pressure } \\
& \text { LV }=\text { left ventricular } \\
& \text { LVAD }=\text { left ventricular assist device } \\
& \text { LVP }=\text { left ventricular pressure } \\
& \text { MAP }=\text { mean arterial pressure } \\
& \text { PVA }=\text { pressure-volume area } \\
& \text { SHE }=\text { surplus hemodynamic energy }
\end{aligned}
$$

blood aspiration and ejection by cyclically actuating a piston around a toroidal pumping chamber via magnetic coupling to a motor while maintaining a second piston in a stationary position to occlude blood flow between the inlet and outlet ports. As the first piston completes the cycle through the torus, a second piston begins a cycle to maintain forward flow while the first piston becomes stationary between the inlet and outlet ports, which maintains unidirectional pulsatile blood flow. No valves are required to produce forward blood flow, there are no areas of stasis, and flow is primarily laminar with very low shear. The current pump can eject up to $37.5 \mathrm{~mL}$ per stroke but can be programmed, using the integrated control module, to eject fractions of the maximum stroke volume. The pump actively aspirates blood from the LV apex and delivers it to the ascending aorta in synchrony with the native cardiac cycle via electrocardiographic triggering. A schematic of the pump is depicted in Figure 1. The pump can also be operated in an asynchronous, continuous mode providing more than 8 liters of forward pulsatile flow/min. A flow dynamic computer simulation suggested that pulsatile flow synchronized to the cardiac cycle would produce superior LV unloading and reduced LV work compared with continuous flow at the same level of support.

There has never been a direct comparison between a positive displacement, pulsatile flow, pump capable of LV assistance synchronized with the cardiac cycle and a continuous-flow LVAD in terms of effectiveness of LV unloading, optimal circulatory support, and reduced myocardial work for a given level of circulatory support. The purpose of this investigation was to test the hypothesis that pulsatile LV assistance, synchronized with the cardiac cycle, provides superior LV unloading and circulatory support when compared with a continuous-flow LV assist device at a similar level of LVAD flow.

\section{MATERIALS AND METHODS}

Because there are no hemodynamic circulatory loops capable of accurately evaluating myocardial work and unloading comparing continuous flow with synchronized pulsatile flow, an animal model was chosen. A total of 16 male domestic farm raised pigs (Yorkshire/Poulin mixed) weighing $63 \mathrm{~kg}(53-73 \mathrm{~kg})$ were used in this study. Five pigs were used to optimize the aortic graft anastomosis and LV inflow cannula implantation location and techniques. Four pigs were used to test the TORVAD pump function and determine optimal timing of synchronization with the cardiac cycle for LV unloading and circulatory support. To minimize the number of animals required for the study, both pumps were inserted in each study animal with each animal serving as its own control. The remaining 7 pigs were used for the final data set comparing the TORVAD with the BioMedicus model BPX-80 centrifugal pump (Medtronic, Inc, Minneapolis, MN). Animals received proper care in compliance with the Animal Welfare Committee of the University of Texas at Houston Medical School, and all experiments were performed according to the Animal Welfare Committee's guidelines.

\section{Surgical Procedure}

Animals were sedated with an intramuscular injection of telazol $4 \mathrm{mg} / \mathrm{kg}$ (Wyeth, Madison, NJ) and robinul (American Regent, Inc, Shirley, NY) $0.0009 \mathrm{mg} / \mathrm{kg}$ and endotracheally intubated. Anesthesia was initiated with $3 \%$ to $5 \%$ isoflurane, and animals were maintained under general anesthesia with isoflurane $2 \%$ to $3 \%$ and mechanically ventilated with oxygen while the body temperature was maintained at $38^{\circ} \mathrm{C}$ with a heating pad. A blood transfusion of up to 1 liter of donor pig blood (Lampire Biological Laboratories, Inc, Pipersville, Pa) was given during the procedure as necessary to replace surgical blood loss, and this was supplemented with normal saline as necessary to ensure adequate volume status. All animals received sodium heparin $150 \mathrm{U} / \mathrm{kg}$ intravenous (IV) bolus, and additional boluses, to maintain an activated clotting time greater than 200 seconds. Before sternotomy, animals were given a loading dose of $5.0 \mathrm{mg} / \mathrm{kg}$ amiodarone (Ben Venue Labs, Inc, Bedford, Ohio) and were maintained on a $1 \mathrm{mg} / \mathrm{kg} / \mathrm{h}$ drip to suppress arrhythmias. An IV lidocaine drip (1 mg/kg/h) was also maintained throughout the procedure with additional boluses of lidocaine (1-2 mg/kg) administered as needed for arrhythmias.

The internal carotid and jugular veins were exposed, and central venous pressure was measured via the left internal jugular vein. A median sternotomy was performed. Left atrial pressure (LAP) was measured with a fluidfilled pressure transducer (Becton Dickinson DTX Plus transducer, ref 682018, Becton Dickinson Infusion Therapy Systems, Sandy, Utah) via a catheter placed in the left atrial appendage. Left ventricular pressure (LVP) was measured with a Millar catheter (model SPR-524 3.5F Mikro-tip Millar Instruments, Inc, Houston, Tex) placed near the LV apex through a $4 \mathrm{~F}$ sheath. Aortic pressure (AoP) was measured with a Millar catheter placed in the ascending aorta. Aortic blood flow was measured with a $20-\mathrm{mm}$ perivascular ultrasonic flow probe at the base of the aorta (MA20PAX, Transonic Systems Inc, Ithaca, NY). LVAD pump blood flow was measured with an ultrasonic flow probe attached to the pump inflow cannula (ME11PXL Transonic Systems Inc, Ithaca, NY). Ventricular volume was indirectly measured using 2 piezo-electric sonomicrometer crystals (Sonometrics Corp, London, Ontario, Canada, and Craig J. Hartley, Baylor College of Medicine, Houston, Tex) sutured on the short axis of the heart at the level of the papillary muscles. The surface electrocardiogram (ECG) was recorded. All measurements were acquired and recorded continuously with a 16-channel PONEMAH physiologic digital data acquisition system (Data Sciences International, St Paul, Minn). Heart failure was induced by ligation of the left anterior descending (LAD) coronary artery after the first diagonal branch with a suture ligature after baseline data were recorded.

The $12.7 \mathrm{~mm}$ inflow cannula was then inserted into the LV apex and secured with horizontal mattress sutures of pledgeted 3-0 Prolene. A 10-mm Dacron graft was then anastomosed to the brachiocephalic artery in an endto-side fashion with 7-0 Prolene while the brachiocephalic artery was occluded proximally and distally. The TORVAD was primed with saline and de-aired. The pump was then connected to the inflow and outflow cannulae via Y-connectors with careful detail given to exclude air bubbles. 


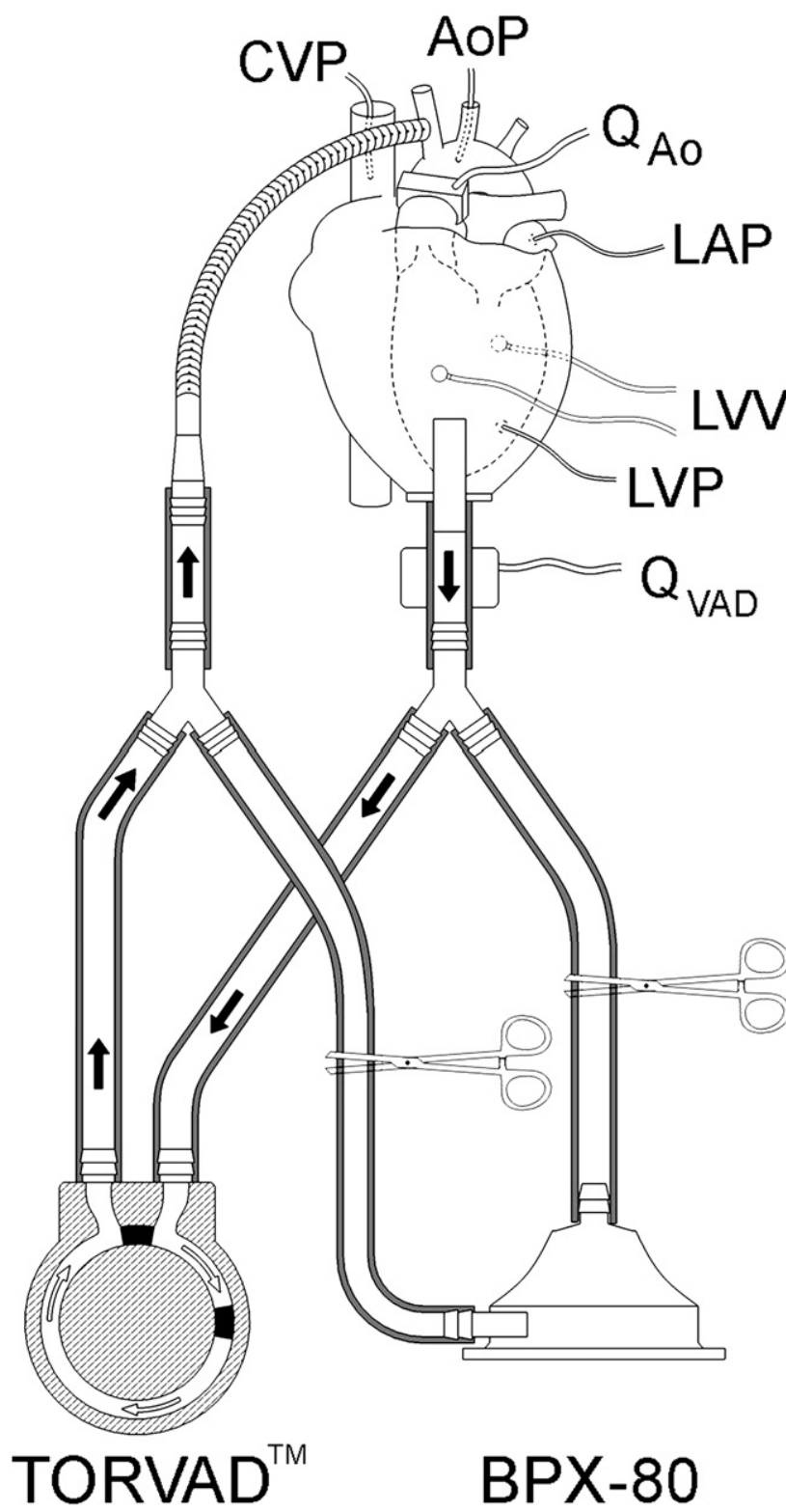

FIGURE 1. Schematic of the experimental preparation and instrumentation. $L V V$, Left ventricular volume crystals; $L V P$, left ventricular pressure micromanometer; $Q_{V A D}$, VAD flow probe; $L A P$, left atrial pressure catheter; $Q_{A o}$, aortic flow probe; $A o P$, aortic pressure micromanometer; $C V P$, central venous pressure catheter.

A BioMedicus BPX 80 pump was then attached to the other end of the Y-connectors in a similar fashion. Either LVAD could be isolated from the circulation with tubing clamps allowing support of the circulation with either LVAD independently. A schematic of the experimental setup is depicted in Figure 1.

\section{Hemodynamic Assessment}

Once surgical implantation of the TORVAD and Bio-Medicus pumps was completed, hemodynamics were assessed in 4 physiologic states: baseline with and without LV assistance and failure after ligation of the midLAD coronary artery with and without LV assistance. The failure model was produced using LAD ligation just distal to the first diagonal branch to induce significant ischemia in the lower left ventricle, reducing LV contractility and lowering cardiac output and mean AoP. The target LVAD flow rate was $37.5 \mathrm{~mL}$ times the heart rate, which was the maximum stroke volume for the TORVAD when activated once during each cardiac cycle. The TORVAD provided synchronous support using epicardial ECG triggering, where a single ejection was performed with each heart beat timed in early diastole. Thus, blood was actively aspirated from the LV apex and simultaneously delivered to the aorta providing an early-diastolic counterpulse (Figure 2,C). This timing maintained the ejection of the native ventricle through the aortic valve during systole (at reduced pre and after-load), which allowed a significant portion of the cardiac output to be sensitive to the physiologic flow regulators of ventricular preload and after-load.

At each physiologic state (baseline off and on support, followed by LAD ischemic failure off and on support), each pump was allowed to run for 5 minutes to allow for stabilization of hemodynamics before beginning a 30-second data-collection period. After data had been collected with 1 pump, the tubing clamps were switched to redirect blood flow and support was established with the other pump. The data gathered included heart rate, cardiac output (aortic plus LVAD flow), LVAD flow, AoP and aortic flow, LVP, LAP, central venous pressure, epicardial ECG, and LV volume, which was calculated from the epicardial diameter crystals. Data were obtained in random order during either TORVAD or BioMedicus LVAD support at the same blood flow rates. Instantaneous LV volume was calculated using a prolate semi-spheroid shell model of the left ventricle ${ }^{9}$ by the formula

$$
\operatorname{LVV}=\frac{\pi}{6}(b-2 h)^{2}(a-1.1 h)
$$

where the long axis $(a)$ and wall thickness $(h)$ were measured postmortem, and the external short axis $(b)$ was continuously obtained using the piezoelectric crystal pair. Pressure-volume loops were then constructed using high-fidelity LVP and the corresponding instantaneous LV volumes.

LVP-volume area (PVA) represents the total mechanical energy of contraction and correlates strongly with LV oxygen consumption. ${ }^{10} \mathrm{LV}$ PVA is the sum of the external mechanical work, which is the area in the LVPvolume loop, and the mechanical potential energy, which is the area contained by the pressure-volume loop, the end-diastolic pressure-volume relationship curve, and the end-systolic pressure-volume relationship curve.

Surplus hemodynamic energy (SHE) is a measure of the extra energy generated by cardiac or LVAD flow pulsation above the mean arterial pressure (MAP) ${ }^{11}$ and quantifies pulsatility. SHE is obtained by subtracting MAP from the energy equivalent pressure, which is calculated by dividing the area beneath the hemodynamic power curve by the area beneath the hemodynamic flow curve

$$
S H E=\frac{\int Q P d t}{\int Q d t}-\mathrm{MAP}
$$

where $Q$ is the total flow rate of the native aortic flow and VAD flow, and $P$ is the arterial pressure.

After the initial measurements in the control state, the LAD was ligated, which resulted in LV ischemia and concomitant failure. After approximately 15 minutes of stable LV failure was achieved, hemodynamic measurements were obtained on and off LVAD support with both pumps sequentially, in random order for each animal. Each animal was then sacrificed with IV supersaturated potassium chloride; the heart was excised from the chest, and LV length and wall thickness measurements were determined for use in the volume calculations as described above.

\section{RESULTS}

Seven animals were instrumented, 1 animal died during instrumentation, and the remaining 6 animals form the basis 

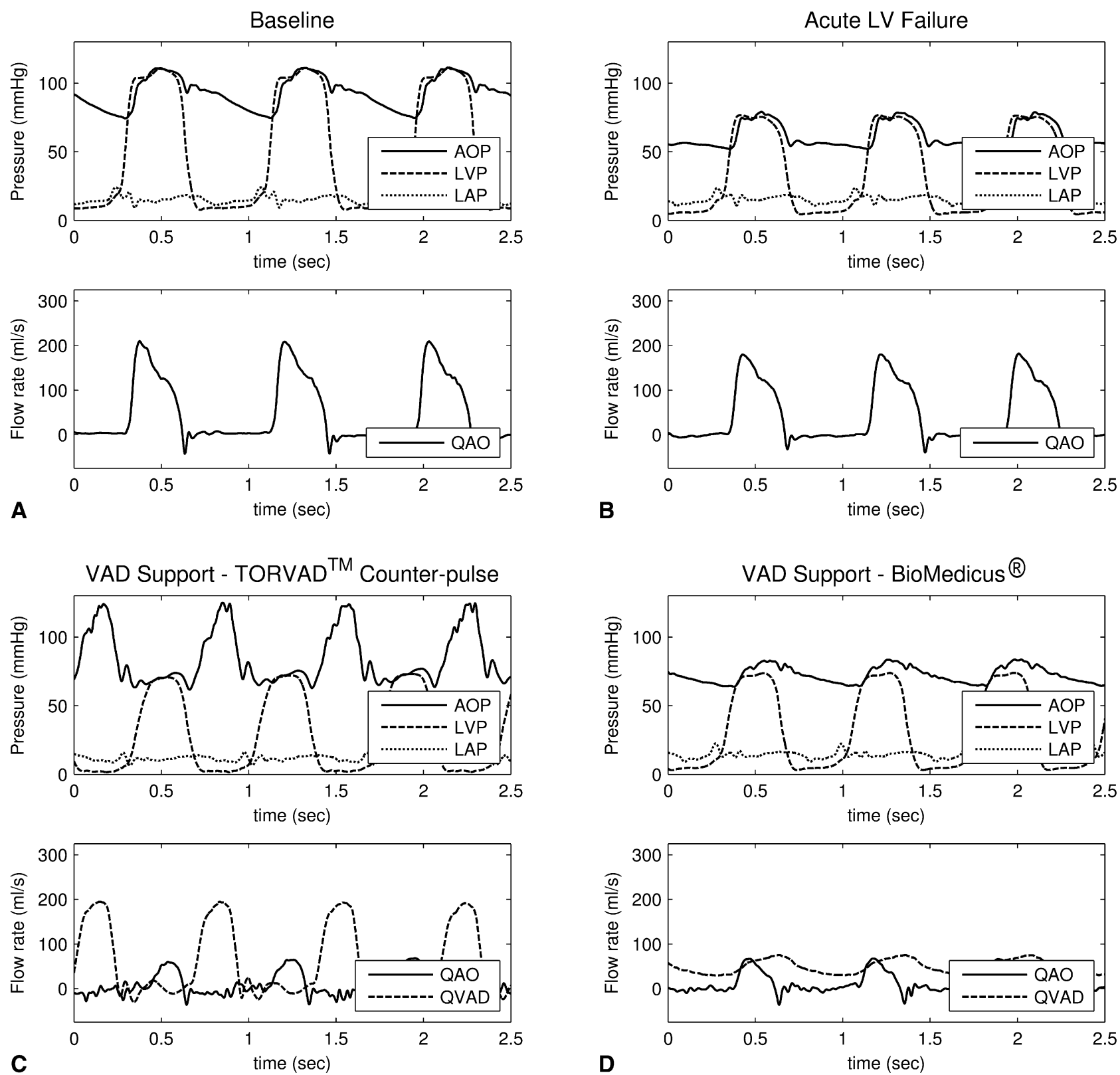

FIGURE 2. Hemodynamic recordings obtained from pig 14 during baseline (A), with LAD ligation and acute LV failure (B), with TORVAD (Windmill Cardiovascular Systems, Inc, Austin, TX) support during failure (C), and with BioMedicus (Medtronic, Inc, Minneapolis, MN) support during failure (D). $A O P$, Aortic pressure; $L V$, left ventricular; $L V P$, left ventricular pressure; $L A P$, left atrial pressure; $Q A O$, transaortic valve flow; $Q V A D$, ventricular assist device output.

of this report. Hemodynamic measurements were obtained with no LV assistance or with either TORVAD support or BioMedicus support initially (randomly assigned). During the data-collection periods, the heart rate remained at approximately 90 beats $/ \mathrm{min}$. All animals were in sinus rhythm and were not paced. As outlined in Table 1, at baseline, the mean AoP was $73 \pm 13 \mathrm{~mm} \mathrm{Hg}$ while the mean LAP was $18 \pm 8 \mathrm{~mm} \mathrm{Hg}$ and the cardiac output was $4.49 \pm 1.1 \mathrm{~L} / \mathrm{min}$. The average hematocrit was $27 \% \pm$ $2 \%$ at the beginning of the procedure pre-sternotomy and
$28 \% \pm 4 \%$ during the period of data collection. All animals were hemodynamically stable during the baseline and the LV failure data-collection periods.

\section{Hemodynamic Effects of Left Ventricular Assistance in the Healthy Animal}

The LVAD flow rate was constant with both devices to ensure the measured results reflect the difference between continuous versus synchronized pulsatile support independently of changes in LVAD flow levels. With initiation of 
TABLE 1. Hemodynamic measurements

\begin{tabular}{|c|c|c|c|c|c|c|}
\hline & \multicolumn{3}{|c|}{ Healthy } & \multicolumn{3}{|c|}{ Ligated } \\
\hline & Baseline & $\begin{array}{c}\text { TORVAD } \\
\text { (Windmill Cardiovascular } \\
\text { Systems, Inc, Austin, Tex) }\end{array}$ & $\begin{array}{l}\text { BioMedicus (Medtronic, } \\
\text { Inc, Minneapolis, Minn) }\end{array}$ & Baseline & TORVAD & BioMedicus \\
\hline HR (bpm) & $90 \pm 16$ & $89 \pm 15$ & $88 \pm 18$ & $86 \pm 12$ & $91 \pm 13$ & $88 \pm 11$ \\
\hline $\mathrm{CO}(\mathrm{L} / \mathrm{m})$ & $4.49 \pm 1.1$ & $5.87 \pm 1.3^{*}$ & $5.42 \pm 1.2$ & $3.54 \pm 1.25$ & $5.58 \pm 1.58 *$ & $5.12 \pm 1.19$ \\
\hline QVAD (L/m) & $0.00 \pm 0.0$ & $3.27 \pm 0.6$ & $3.27 \pm 0.71$ & $0.00 \pm 0.00$ & $3.39 \pm 0.50$ & $3.36 \pm 0.56$ \\
\hline QAo & $4.49 \pm 1.1$ & $2.60 \pm 0.9^{*}$ & $2.15 \pm 0.84$ & $3.54 \pm 1.25$ & $2.19 \pm 1.36^{*}$ & $1.77 \pm 0.95$ \\
\hline AoPmax $(\mathrm{mm} \mathrm{Hg})$ & $96.0 \pm 9.0$ & $100.5 \pm 18.8^{*}$ & $92.0 \pm 9.0$ & $75.8 \pm 15.9$ & $89.1 \pm 23.8^{*}$ & $74.9 \pm 14.7$ \\
\hline AoPmin $(\mathrm{mm} \mathrm{Hg})$ & $56.7 \pm 11.8$ & $62.7 \pm 18.5$ & $61.5 \pm 9.6$ & $41.8 \pm 11.8$ & $48.9 \pm 12.8$ & $51.7 \pm 8.8$ \\
\hline AoPavg (mm Hg) & $73.1 \pm 12.5$ & $82.0 \pm 17.3^{*}$ & $74.2 \pm 11.6$ & $54.6 \pm 12.3$ & $67.8 \pm 14.0 *$ & $60.2 \pm 10.0$ \\
\hline LAPavg (mm Hg) & $18.4 \pm 8.0$ & $11.6 \pm 2.7^{*}$ & $13.2 \pm 3.7$ & $18.9 \pm 8.0$ & $11.5 \pm 3.4^{*}$ & $13.9 \pm 6.0$ \\
\hline PVA $(\mathrm{mL} \mathrm{mm} \mathrm{Hg})$ & $6945 \pm 1972$ & $5697 \pm 1382$ & $6254 \pm 1901$ & $4926 \pm 1114$ & $4229 \pm 1179 \dagger$ & $4560 \pm 125$ \\
\hline $\mathrm{SHE}(\mathrm{mm} \mathrm{Hg})$ & $14.3 \pm 3.2$ & $7.4 \pm 6.7$ & $5.9 \pm 1.3$ & $13.5 \pm 2.3$ & $7.6 \pm 6.4$ & $3.8 \pm 2.2$ \\
\hline
\end{tabular}

$H R$, Heart rate; $C O$, cardiac output; $Q V A D$, ventricular assist device flow; $Q A o$, aortic valve flow; AoPmax, maximum aortic pressure; $A o P m i n$, minimum aortic pressure; $A o P a v g$, average aortic pressure; $L A P a v g$, average left atrial pressure; $P V A$, pressure-volume area; $S H E$, surplus hemodynamic energy. $* P<.05$ for TORVAD vs BioMedicus. $\nmid P<.10$ for TORVAD vs BioMedicus.

pulsatile LV support with the TORVAD, the cardiac output increased from $4.49 \pm 1.1 \mathrm{~L} / \mathrm{min}$ to $5.87 \pm 1.3 \mathrm{~L} / \mathrm{min}$ (Table 1), which was higher than during BioMedicus continuous-flow support $(5.42 \pm 1.2 \mathrm{~L} / \mathrm{min}, P<.05)$. Both the maximum and the average AoP were elevated with TORVAD support compared with both BioMedicus support and at baseline. In addition, LAP was reduced during TORVAD support and was lower than during BioMedicus support $(11.6 \pm 2.7 \mathrm{~mm} \mathrm{Hg}$ vs $13.2 \pm 3.7 \mathrm{~mm} \mathrm{Hg}$, $P<.05)$.

\section{Hemodynamic Effects of Left Ventricular Assistance During Acute Ischemic Failure}

After ligation of the LAD just distal to the first diagonal branch, the ventricle dilated in the anterolateral and apical regions and the regional wall motion became akinetic by visual inspection. The animal's hemodynamics were allowed to stabilize, and although the heart rate did not change significantly, the mean AoP decreased from $73 \pm 12.5 \mathrm{~mm} \mathrm{Hg}$ to $54.6 \pm$ $12.3 \mathrm{~mm} \mathrm{Hg}$ (See Table 1 and Figure 3). With TORVAD support, the cardiac output increased from $3.54 \pm 1.25 \mathrm{~L} / \mathrm{min}$ to
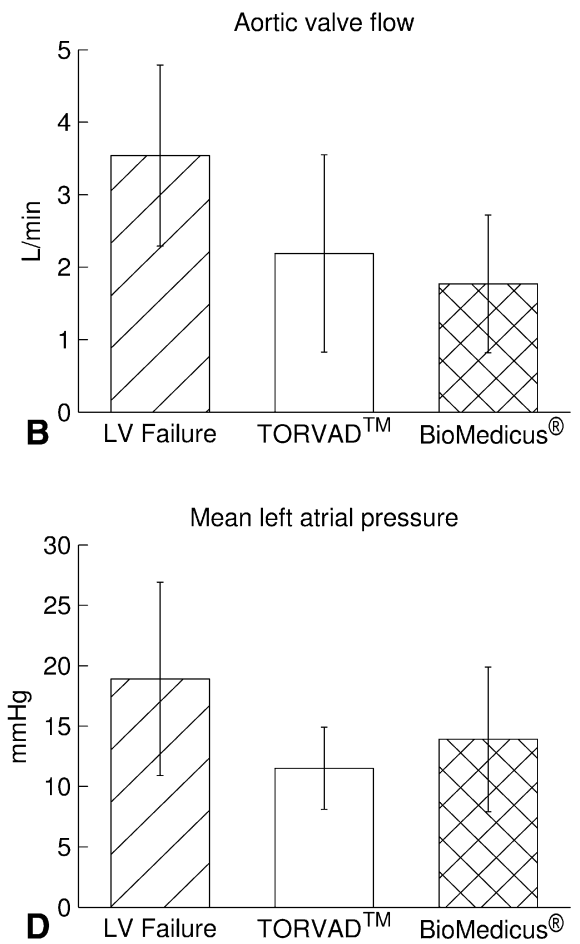

FIGURE 3. Cardiac output (A), aortic valve flow (B), mean LAP (C), and MAP(D) with LAD ligation during acute LV failure. Measurements were obtained during LV failure after LAD occlusion and then with BioMedicus and TORVAD support in random sequence. 


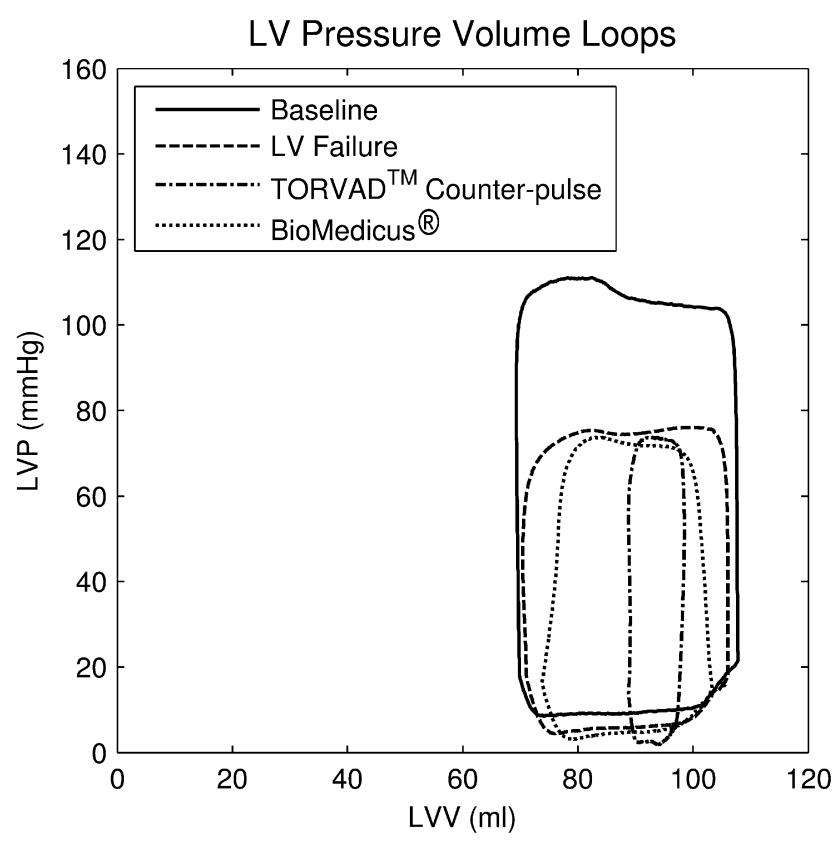

FIGURE 4. Pressure-volume loops obtained from pig 14 during baseline, with LAD ligation and acute LV failure, with BioMedicus support during failure, and with TORVAD support during failure. $L V$, Left ventricular; $L V P$, left ventricular pressure; $L V V$, left ventricular volume.

$5.58 \pm 1.58 \mathrm{~L} / \mathrm{min}$, which was higher than during BioMedicus support $(P<.05)$. With TORVAD support, aortic flow was reduced compared with baseline but remained higher than aortic flow during BioMedicus support $(2.19 \pm 1.36 \mathrm{~L} / \mathrm{min}$ vs $1.77 \pm 0.95 \mathrm{~L} / \mathrm{min}, P<.05)$. MAP increased in TORVAD supported animals to $67.8 \pm 14 \mathrm{~mm} \mathrm{Hg}$ compared with $60.2 \pm$ $10 \mathrm{~mm} \mathrm{Hg}$ with BioMedicus support $(P<.05)$. Similarly, there was a reduction in LAP to $11.5 \pm 3.5 \mathrm{~mm} \mathrm{Hg}$ in TORVAD-supported animals compared with $13.9 \pm 6 \mathrm{~mm}$ $\mathrm{Hg}$ in BioMedicus-supported animals $(P<.05)$.

The differences between synchronized pulsatile and continuous-flow support hemodynamics are illustrated by Figure 2. During LAD ischemia in pig 14 , a decrease in mean AoP from $90 \mathrm{~mm} \mathrm{Hg}$ to $62 \mathrm{~mm} \mathrm{Hg}$ (Figure 2, $A, B$ ) occurred similar to that observed in the other animals. With TORVAD support, a distinct increase in aortic diastolic pressure occurred compared with the BioMedicus device (Figure 2, $C, D$ ). Once again, the LAP diminished significantly in the TORVAD animals $(11.8 \mathrm{~mm} \mathrm{Hg})$ compared with the BioMedicus animals $(14.4 \mathrm{~mm} \mathrm{Hg})$.

\section{Effect of Pulsatile Compared With Continuous-Flow Left Ventricular Assist on Left Ventricular Work}

The PVA serves as an index of LV work, which is depicted graphically with pig 14 data (Figure 4). With initiation of LV failure, the LV generated less work. With BioMedicus continuous-flow support, as shown in the small dotted line in Figure 4, the PVA was reduced further; however, with initiation of TORVAD support, the PVA was reduced dramatically as depicted by the dotted line in Figure 4 . In the aggregate data (Table 1), during ischemic heart failure, the LVP volume area was reduced from $4926 \pm 114 \mathrm{~mL} \mathrm{~mm} \mathrm{Hg}$ to $4229 \pm 1179 \mathrm{~mL} \mathrm{~mm} \mathrm{Hg}$ with pulsatile LV support.

\section{Impact of Pulsatile Compared with Continuous-Flow Left Ventricular Assist on Arterial Pulsatility}

SHE is an index of pulsatility of the AoP. With pulsatile LV support, the SHE was approximately twice that observed during continuous-flow LV assist $(7.6 \pm 6.4 \mathrm{~mm} \mathrm{Hg}$ vs $3.8 \pm 2.2 \mathrm{~mm} \mathrm{Hg}$ ). The diastolic augmentation of AoP during TORVAD counterpulse synchronized support (active VAD flow from LV apex to aorta) was greater than continuous-flow support with the BioMedicus pump (Figure 2, C, D). During ischemic failure in pig 14, the SHE increased from $12 \mathrm{~mm} \mathrm{Hg}$ at baseline to $18 \mathrm{~mm} \mathrm{Hg}$ with synchronized pulsatile LV support compared with $2.4 \mathrm{~mm} \mathrm{Hg}$ with continuous-flow support.

\section{DISCUSSION}

The Interagency Registry for Mechanically Assisted Circulatory Support reported results in 483 patients implanted with mechanical support devices primarily as bridge to transplant (BTT). ${ }^{12}$ Over an 18 -month period, in patients implanted with LVADs, the survival was similar between BTT and destination therapy at approximately $60 \%$ to $70 \%{ }^{12}$ Although the earlier Randomized Evaluation of Mechanical Assistance for the Treatment of Heart Failure Trial (REMATCH) demonstrated improved outcomes in transplantineligible patients treated with a pulsatile LVAD compared with optimal medical therapy, the LVAD failure rate was disappointing. ${ }^{13}$ In contrast, Long and colleagues, ${ }^{14}$ in a study of 23 patients treated with a similar pulsatile-flow LVAD (HeartMate model XVE, Thoratec Corp, Pleasanton, CA) for destination therapy, reported a 77\% 2-year survival compared with $29 \%$ in the REMATCH trial. The HeartMate II (Thoratec Corp, Pleasanton, Calif) investigators, in a BTT population, reported that the continuous-flow HeartMate II device resulted in an 18-month survival of $72 \%{ }^{4}$ with a device failure rate lower than that observed in the REMATCH trial, and the major causes of death included sepsis and stroke ${ }^{4}$ rather than device failure.

The recently reported randomized trial comparing the HeartMate XVE pulsatile device and the HeartMate II continuous-flow device demonstrated that the continuousflow LVAD produced superior survival compared with the pulsatile-flow device. This was due to improved HeartMate II device longevity rather than the physiologic benefits of continuous flow. ${ }^{15}$

No previously reported comparisons of pulsatile flow synchronized to the cardiac cycle and continuous-flow LV support at the same level of LVAD flow exist. In a recent study of 
continuous-flow versus pulsatile-flow devices in patients with decreased renal function at device implant, there was a distinct trend toward improved creatinine clearance in the pulsatile-flow group. ${ }^{16}$ In a study comparing equal numbers of patients on continuous-flow and pulsatile-flow devices for 12 months or longer, a significant improvement in LV remodeling and function was observed with pulsatile-flow LVAD support. ${ }^{17}$ In the pulsatile-flow group, the LV enddiastolic diameter decreased from 77 to $60 \mathrm{~mm}$ compared with $69 \mathrm{~mm}$ in the continuous-flow group $(P=.012)$. Similarly, the ejection fraction improved from $16 \%$ to $20 \%$ in the pulsatile-flow group compared with no change in the continuous-flow group $(P=.022) .{ }^{17}$ Only pulsatile support has demonstrated reductions in ventricular mass. ${ }^{18}$

Travis and colleagues ${ }^{19}$ evaluated SHE in patients on continuous-flow or pulsatile-flow LVAD support. At low support levels, SHE was restored to within $2.5 \%$ normal with pulsatile support but continuous-flow support diminished SHE. At high support levels, SHE increased 49\% with pulsatile flow, whereas continuous flow diminished SHE by $97 \%$. In addition, pulsatile support was associated with normal vascular compliance, whereas continuousflow support resulted in stiffening of the vasculature. ${ }^{19}$

Blood at low shear rates has viscoelastic properties. With increased pulsatility, the viscoelastic interaction of the erythrocytes is reduced, which in turn reduces viscosity and therefore blood flow resistance in the tissue beds. ${ }^{20}$ As a result, tissue oxygen delivery is enhanced in the gut, ${ }^{21}$ renal circulation, ${ }^{22}$ and brain ${ }^{23}$ during circulatory bypass with pulsatile flow compared with continuous flow.

In patients with severe aortic stenosis, small and large bowel arteriovenous malformations are present, which tend to bleed spontaneously. In addition, acquired Von Willebrand disease seems to develop in these patients. ${ }^{24}$ These findings have been suggested as potential causative mechanisms for the apparent increase in gastrointestinal bleeding observed in patients with continuous axial flow LVADs compared with little or no increased gastrointestinal bleeding with pulsatile LVADs. ${ }^{24}$ We previously reported near-fatal gastrointestinal bleeding associated with continuous-flow LVADs, which resolved after cardiac transplant and explantation of the VAD. ${ }^{25}$

\section{Study Limitations}

This acute experiment was performed in highly instrumented, anesthetized animals tested over relatively brief periods of time. Because of the coronary blood flow occurring below the aortic flow probe, using aortic flow for the cardiac output calculation might be slightly in error. It would also have been desirable to have determined beneficial improvements of end-organ perfusion with synchronized pulsatile flow. In controlled experimental conditions, synchronization with the cardiac cycle was feasible. However, in the clinical environment, in the presence of dysrhythmias, synchroniza- tion may be more challenging. Although these results are compelling, further investigations in chronic animal implants, and ultimately in humans, will be necessary to determine the true merit of a reliable pulsatile-flow LVAD that can be synchronized to the cardiac cycle, particularly with regard to potentially improved LV remodeling.

\section{CONCLUSIONS}

Synchronized, pulsatile LV assistance is more physiologic and produces superior LV unloading compared with continuous-flow LV assistance. We have demonstrated improved pulsatility (SHE), reduced LV work, reduced LAP, and improved cardiac output and AoP with pulsatile flow synchronized with the cardiac cycle in early diastole compared with continuous flow with the BioMedicus pump at identical levels of LVAD flow. Programmable, pulsatileflow LVADs with controllable stroke volumes and pumping characteristics may provide attractive devices, particularly for bridge-to-recovery applications in patients with severe heart failure.

The authors thank Cezar Iliescu, MD, Lisa M. Janowiak, BS, CCP, Erik R. Larson, MS, Patricia Felli, BS, James Amirian, BS, and Gerald Dorros, MD, whose help in experimental design, performance, and analysis of the data was critical for its completion.

\section{References}

1. Christiansen S, Klocke A, Autschbach R. Past, present, and future of long-term mechanical cardiac support in adults. J Card Surg. 2008;23:664-76.

2. Potapov EV, Loforte A, Weng Y, Jurmann M, Pasic M, Drews T, et al. Experience with over 1000 implanted ventricular assist devices. J Card Surg. 2008;23 185-94.

3. Miller LW, Pagani FD, Russell SD, John R, Boyle AJ, Aaronson KD, et al. Use of a continuous-flow device in patients awaiting heart transplantation. $N$ Engl J Med. 2007;357:885-96.

4. Pagani FD, Miller LW, Russell SD, Aaronson KD, John R, Boyle AJ, et al Extended mechanical circulatory support with a continuous-flow rotary left ventricular assist device. J Am Coll Cardiol. 2009;54:312-21.

5. Klotz S, Jan Danser AH, Burkhoff D. Impact of left ventricular assist device (LVAD) support on the cardiac reverse remodeling process. Prog Biophys Mol Biol. 2008;97:479-96.

6. Letsou GV, Connelly JH, Delgado RM 3rd, Myers TJ, Gregoric ID, Smart FW, et al. Is native aortic valve commissural fusion in patients with long-term left ventricular assist devices associated with clinically important aortic insufficiency? J Heart Lung Transplant. 2006;25:395-9.

7. Meyer AL, Kuehn C, Weidemann J, Malehsa D, Bara C, Fischer S, et al. Thrombus formation in a HeartMate II left ventricular assist device. J Thorac Cardiovasc Surg. 2008;135:203-4.

8. Myers TJ, Bolmers M, Gregoric ID, Kar B, Frazier OH. Assessment of arterial blood pressure during support with an axial flow left ventricular assist device. J Heart Lung Transplant. 2009;28:423-7.

9. Field S, Drzewiecki G. Real-time left ventricular volume of the canine heart from ultrasonic dimension data. IEEE Trans Biomed Eng. 1993;40:1070-3.

10. Khalafbeigui F, Suga H, Sagawa K. Left ventricular systolic pressure-volume area correlates with oxygen consumption. Am J Physiol. 1979;237:H566-9.

11. Undar A, Zapanta CM, Reibson JD, Souba M, Lukic B, Weiss WJ, et al. Precise quantification of pressure flow waveforms of a pulsatile ventricular assist device. ASAIO J. 2005;51:56-9.

12. Holman WL, Pae WE, Teutenberg JJ, Acker MA, Naftel DC, Sun BC, et al INTERMACS: interval analysis of registry data. J Am Coll Surg. 2009;208:755-62.

13. Rose EA, Gelijns AC, Moskowitz AJ, Heitjan DF, Stevenson LW, Dembitsky W, et al. Long-term mechanical left ventricular assistance for end-stage heart failure. N Engl J Med. 2001;345:1435-43. 
14. Long JW, Healy AH, Rasmusson BY, Cowley CG, Nelson KE, Kfoury AG, et al. Improving outcomes with long-term "destination"' therapy using left ventricular assist devices. J Thorac Cardiovasc Surg. 2008;135:1353-61.

15. Slaughter MS, Rogers JG, Milano CA, Russel SD, Conte JV, Feldman D, et al. Advanced heart failure treated with continuous-flow left ventricular assist device. N Engl J Med. 2009;361:2241-51. Epub 2009 Nov 17.

16. Kamdar F, Boyle A, Liao K, Colvin-adams M, Joyce L, John R. Effects of centrifugal, axial, and pulsatile left ventricular assist device support on end-organ function in heart failure patients. J Heart Lung Transplant. 2009;28:352-9.

17. Drews T, Jurmann M, Michael D, Miralem P, Weng Y, Hetzer R. Differences in pulsatile and non-pulsatile mechanical circulatory support in long-term use. J Heart Lung Transplant. 2008;27:1096-101.

18. Thohan V, Stetson SJ, Nagueh SF, Rivas-Gotz C, Koerner MM, Lafuente JA, et al. Cellular and hemodynamics responses of failing myocardium to continuous flow mechanical circulatory support using the DeBakey-Noon left ventricular assist device: a comparative analysis with pulsatile-type devices. J Heart Lung Transplant. 2005;24:566-75.

19. Travis AR, Giridharan GA, Pantalos GM, Dowling RD, Prabhu SD, Slaughter MS, et al. Vascular pulsatility in patients with a pulsatile- or continuous-flow ventricular assist device. J Thorac Cardiovasc Surg. 2007;133:517-24.
20. Undar A, Henderson N, Thurston GB, Masai T, Beyer EA, Frazier OH, et al. The effects of pulsatile versus nonpulsatile perfusion on blood viscoelasticity before and after deep hypothermic circulatory arrest in a neonatal piglet model. Artif Organs. 1999;23:717-21.

21. Ohri SK, Bowles CW, Mathie RT, Lawrence DR, Keogh BE, Taylor KM. Effect of cardiopulmonary bypass perfusion protocols on gut tissue oxygenation and blood flow. Ann Thorac Surg. 1997;64:163-70.

22. Kim HK, Son HS, Fang YH, Park SY, Hwang CM, Sun K. The effects of pulsatile flow upon renal tissue perfusion during cardiopulmonary bypass: a comparative study of pulsatile and nonpulsatile flow. ASAIO J. 2005;51:30-6.

23. Undar A, Eichstaedt HC, Frazier OH, Fraser CD Jr. Monitoring regional cerebral oxygen saturation using near-infrared spectroscopy during pulsatile hypothermic cardiopulmonary bypass in a neonatal piglet model. ASAIO J. 2000;46:103-6.

24. Crow S, John R, Boyle A, Shumway S, Liao K, Colvin-Adams M, et al. Gastrointestinal bleeding rates in recipients of nonpulsatile and pulsatile left ventricular assist devices. J Thorac Cardiovasc Surg. 2009;137:208-15.

25. Letsou GV, Shah N, Gregoric ID, Myers TJ, Delgado R, Frazier OH. Gastrointestinal bleeding from arteriovenous malformations in patients supported by the Jarvik 2000 axial-flow left ventricular assist device. J Heart Lung Transplant. 2005;24:105-9. 